\title{
The safety and efficacy of fecal microbiota transplantation in a population with bipolar disorder during depressive episodes: study protocol for a pilot randomized controlled trial
}

Noah C. A. Cooke ${ }^{1}$, Asem Bala ${ }^{2}$, Johane P. Allard ${ }^{3}$, Susy Hota ${ }^{4}$, Susan Poutanen ${ }^{5}$ and Valerie H. Taylor ${ }^{2,6^{*}}$ (D)

\begin{abstract}
Background: Bipolar disorder (BD) is a chronic, debilitating illness with significant medical morbidity, often secondary to current treatments, and a high recurrence rate. This burden of disease reflects limitations in the tolerability and efficacy of current treatments. There is a compelling body of evidence linking the gut microbiota to mental illness, and while microbial manipulation via probiotic use has been studied as a therapeutic in BD, targeted trials of fecal microbiota transplantation (FMT) have not been conducted in this population.

Methods and design: We describe a pilot randomized controlled trial of FMT in participants with BD depression to assess the feasibility, efficacy, safety, and tolerability of this intervention. Individuals between 18 and 65 years of age will be enrolled in the study if they meet diagnostic criteria for a major depressive episode of at least moderate severity in the context of a BD diagnosis and have not responded to treatment for BD. Participants will be randomized 1:1 to receive either screened and processed donor stool (allogenic FMT) or their own stool (autologous FMT) via colonoscopy and monitored for 24 weeks post intervention. Depressive and manic symptoms, treatment acceptability, and gastrointestinal and other side effects are assessed at baseline (prior to randomization) and weekly. Stool samples to assess microbiome composition are obtained at baseline and 3 and 6 months.

Discussion: Currently, FMT represents a novel therapeutic option for treating BD depression. This protocol allows for the assessment of the feasibility, efficacy, acceptability, and safety of an intervention aimed at changing the microbiome in those with $\mathrm{BD}$. Results from this pilot study will guide the development of larger trials of FMT for BD depression and may give more insight into how the gut microbiome are altered in those with BD depression.
\end{abstract}

Trial registration: Clinical Trials Gov NCT03279224

Keywords: FMT, Bipolar disorder, Microbiome, Mood

\footnotetext{
* Correspondence: valerie.taylor@ucalgary.ca

${ }^{2}$ Department of Psychiatry, Cumming School of Medicine, University of Calgary, Calgary, Alberta, Canada

${ }^{6}$ Hotchkiss Brain Institute, Cumming School of Medicine, University of Calgary, Calgary, Alberta, Canada

Full list of author information is available at the end of the article
} 


\section{Background}

Bipolar disorder (BD) is a chronic and debilitating condition with an estimated lifetime prevalence of over $2.0 \%$ [1]. While the illness is characterized in part by recurring episodes of mania or hypomania, depression accounts for up to $72 \%$ of time during which a person is ill [2]. The depressive components of the disorder are also especially challenging to treat and account for clinically significant residual morbidity [3]. A recent review of treatments found no high or moderate strength of evidence for any treatment of the depressive phase of this BD depression [4], supporting research into alternative therapeutic options.

Mounting evidence suggests that the MGB-axis, comprised of neural, humoral, and cellular routes connecting the gut microbiota with the central nervous system (CNS), is perturbed in neuropsychiatric disorders [5]. Essentially, the gut microbiota is able to modulate the MGB-axis both directly and indirectly via endocrine, neural, and immune pathways. In disease or stress states, these pathways may become dysregulated, resulting in intestinal dysbiosis, changes in mood, behavior, and cognition, and altered inflammatory levels [6]. Specific alterations in gut microbiome composition have been identified in individuals with $\mathrm{BD}$ [7-10] and correlate with illness severity [11]. These findings provide a compelling rationale to investigate whether microbiota-targeted interventions have potential for the treatment of BD.

Several strategies for restoring the MGB-axis to a healthy state have been explored in the treatment of psychiatric disorders. As opposed to short-term effects on the microbiome via probiotic [12] and antibiotic administration [13], it is the use of fecal microbiota transplantation (FMT) that may offer the best biotherapeutic option. It increases microbial diversity and does not result in microbiota dysbiosis as does antibiotic treatment. Unlike probiotics, whose colonization appears to be transient, FMT also affords long-term engraftment of the donor strains [14].

To date, no clinical trials have investigated the feasibility and efficacy of FMT in individuals with BD, but a recent systematic review of preclinical and clinical work reported a decrease in depressive and anxiety-like symptoms and behaviours resulting from the transplantation of healthy microbiota, providing support for this area of research [15]. Herein, we present the methodology of a pilot randomized controlled study that evaluates the feasibility of a trial protocol for FMT in participants with BD depression.

The primary objective of this protocol is to evaluate the feasibility of FMT in a BD population and to also examine FMT efficacy in reducing depression severity in BD patients on stable, first-line treatment for BD depression with a prospective, double-blind randomized controlled trial (RCT). This will guide the development of a larger multisite RCT and further our understanding of the potential biotherapeutic potential of FMT. Other secondary objective of the pilot study are to determine the acceptability, safety, and tolerability of FMT in this population.

\section{Methods}

\section{Trial design}

This study is a phase $2 / 3$, double-blind, placebocontrolled RCT (See Fig. 1). Patients with BD on stable, first-line treatment will be randomized to receive either (1) allogenic FMT from a healthy donor or (2) autologous FMT with their own feces. After screening, FMT will be manufactured into an enema and administered via colonoscopy by a trained gastroenterologist. Participants will then be followed for 24 weeks for assessment of primary and secondary study outcomes. We plan to recruit 60 patients: 30 each for the intervention and control groups. This protocol is reported as per the SPIRIT guidelines [16].

The study will be conducted at the Psychiatry Department at Women's College Hospital (Research Ethics Board [REB] \# 2017-0099-B), with FMT preparation occurring in the University Health Network/Sinai Health System Department of Microbiology (REB\# 17-0289-E) and FMT administration occurring at the endoscopy unit at University Health Network, Toronto Western Hospital (REB\#175147). All hospital sites are affiliated with the University of Toronto. The study received a No Objection Letter from Health Canada (HC6-0240C201262). To help achieve adequate participant enrolment, flyers have been placed at the recruitment sites, and physicians providing clinical care at these sites have been informed of the study to assist with identification of potentially eligible participants. Physician education about the study has included small and large group presentations, as well as an information sheet.

The active phase of the study involves administration of 1 allogenic or autologous FMT course. Participants are then followed for 24 weeks, with stool samples taken and stored at baseline and 3 and 6 months. All study questionnaires will also be administered at each visit as well as review of side effects experienced. Outcome data collectors, participants, and physicians providing clinical care are blind to study group allocation.

The study is registered with ClinicalTrials.Gov under the identifier NCT02116127.

\section{Eligibility criteria}

Eligible participants must be outpatients between 18 and 65 years of age, have a diagnosis of BD (type I or II) according to the Mini International Neuropsychiatric Interview (MINI) [17], have been on a stable first-line treatment for $\mathrm{BD}$ depression as defined in the Canadian 


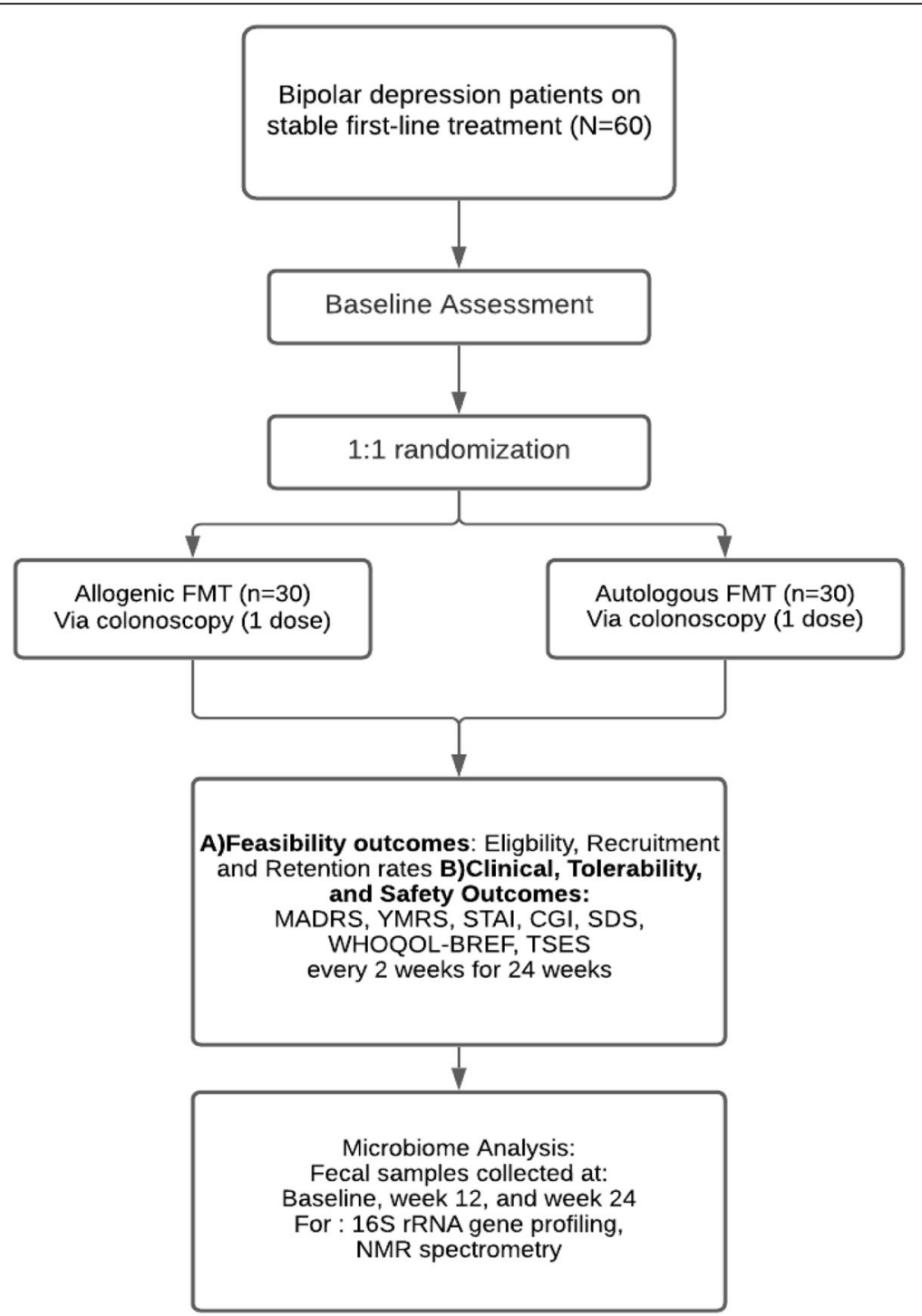

Fig. 1 Trial design. Randomization schema of a pilot randomized controlled trial on the feasibility, safety and efficacy of FMT for Bipolar Disorder. FMT, fecal microbiota transplant; MADRS, Montgomery-Asberg Depression Rating Scale; YMRS, Young Mania Rating Scale; STAI, State Trait Anxiety Index; CGI, Clinical Global Impressions; SDS, Sheehan Disability Scale; WHOQOL, World Health Organization Quality of Life; TSES, Toronto Side Effect Scale

Network for Mood an Anxiety Treatment (CANMAT) 2018 guidelines [18] at an adequate dose for $\geq 8$ weeks prior to study entry, and currently be suffering from a depressive episode, characterized by a Montgomery-Asberg Depression Rating Scale (MADRS) [19] score at screening and baseline of $\geq 20$. Patients will be excluded if they have a Young Mania Rating Scale (YMRS) [20] score of $\geq 12$ at screening; meet DSM-IV criteria [21] for substance abuse within the last 6 months or lifetime dependency; have an active eating disorder; have schizophrenia or schizoaffective disorder; have chronic gastrointestinal diseases; conditions causing immunosuppression; a significant bleeding disorder; a history of incomplete colonoscopy; are pregnant or breastfeeding; have regularly used non-steroidal anti-inflammatory drugs, antibiotics, or iron supplements within 3 months prior to study entry; have used prebiotics or probiotics for medical purposes, or have taken antibiotics or any experimental drug within 3 months prior to study entry.

The healthy donors who provided the allogenic FMT will be recruited as part of the Microbial Therapeutics Outcome Program (MTOP) at University Health Network, Sinai Health System and University of Toronto. Potential donors will be excluded if they have a psychiatric disorder according to the MINI [17], a family history in first-degree (blood) relatives of psychiatric illness, a personal or family history of autoimmune disease in firstdegree (blood) relatives; anaphylactic food or environmental allergies; diabetes or "pre-diabetes", defined as HbA1c $>6 \%$; are a smoker (cigarette, marijuana, other); use 
recreational drugs; regularly consume 2 or more alcoholic beverages daily; and regularly use iron supplements in the 3 months prior to study entry; have a history or symptoms suggestive of underlying gastrointestinal disease; infection or colonization with transmissible agents for repeated donation; active malignancy, or any cancer within the last 5 years, excluding basal cell carcinoma of the skin; risk factors for prion-related disease, including family history of Creutzfeld-Jacob Disease, corneal or dural transplant, or receipt of human-derived pituitary growth factor; history or high-risk status for recent acquisition of HIV, Hepatitis B, or Hepatitis C or sexually transmitted illnesses; immunosuppression; history or physical findings of chronic liver disease or cholestasis; evidence of active encephalitis or meningitis; evidence of active systemic viral, bacterial or fungal infection; receipt of live vaccine in preceding 30 days; receipt of blood transfusion from a country other than Canada in preceding 6 months; history of dementia or degenerative neurological disorders of unknown etiology; recent bite from an animal that may have rabies within the past 6 months; antibiotic use in the 6 months preceding donation; probiotic use for medicinal purposes in the 3 months prior to donation; use of cholestyramine within 3 months of donation; known and current history of blood in stools; and travel outside Canada or the USA in the past 6 months.

To ensure that recipients with severe or anaphylactic reactions to specific foods do not receive FMT from a donor who has recently consumed those foods, dietary screening utilizing a questionnaire developed by the MTOP program will be performed along with the regular prospective screening for medical health, travel and other relevant exposures using a brief checklist for consumption of common allergens. Detailed medical assessment will also be conducted to ensure potential donors are at low risk of infectious disease. This will be further confirmed by stool analysis, free from infectious agents.

\section{Recruitment}

Potentially eligible participants who are willing to hear more about the study receive a detailed study explanation from the study research coordinator. Subjects are provided with a clear explanation of the objectives, procedures, risks, and benefits of the study, and all questions are answered. Questions are asked of subjects to ensure that they understand the nature of the research, risks, and potential benefits of study participation, and their rights as research subjects prior to their signing the informed consent document. The study coordinator will obtain informed, written consent during Visit 1, at which time further screening will be performed. The same qualified team members will enroll participants and stool donors. These individuals will not be involved in the clinical care of the patients. To maximize retention, patients will receive reminders of appointments via phone. If a patient misses an appointment, the reason will be investigated, and two attempts will be made to reach them by phone. Participants are given meaningful opportunities during the study to provide ongoing consent for continuation within the study protocol.

\section{Allocation of interventions}

After informed consent is obtained, participants will be randomized to intervention and placebo control groups $1: 1$, according to a blocked randomization list (4 patients per block), generated in SAS proc PLAN by an individual at the University Health Network not involved in conducting or analyzing the study. Both the participant and study team, except for the lab technologist, will be blinded to group interventions. The allocation list will be kept in sequentially numbered envelopes with the group assignments. The lab technologist will maintain allocation concealment and prepare fecal filtrates for each patient but not be involved in any other part of the study. The researcher performing FMT will receive the designated fecal filtrate labeled only with the participants' numbers. If an adverse event occurs that is thought to be potentially linked to FMT and requires immediate investigation, the code will be broken only for the affected patient. Only the study physician with the applicable expertise will be unblinded; the rest of the study team will not be informed.

\section{Intervention}

Each participant receives one dose of either autologous or allogenic FMT product. FMT manufacturing will be performed as in a previous RCT (NCT01226992). Briefly, $50 \mathrm{~g}$ of screened donor or participant feces will be homogenized for 30 min using a Stomacher ${ }^{\circ}$ Paddle Blender with $30 \mathrm{~mL}$ sterile $0.9 \% \mathrm{NaCl}+10 \%$ glycerol in a sterile, 330- $\mu$ n microfilter-separated, doublecompartment polyethylene bag. The autologous FMT control arm is essential because bowel preparation and colonoscopy can induce changes to the intestinal microbiome [22, 23]. The volume of fecal filtrate corresponding to $50 \mathrm{~g}$ of original feces will be aliquoted, labeled with a recipient ID number to maintain blinding, and stored at $-80{ }^{\circ} \mathrm{C}$. Freezing stool with glycerol as a cryoprotectant has been shown to yield active FMT preparations [24, 25]. For instance, frozen fecal filtrate for treatment of Clostridium difficile infection can be stored for at least 6 months in 10\% glycerol without loss of clinical efficacy or viability of the six bacterial groups measured [26]. Similarly, clinical efficacy in treating $C$. difficile infection was shown not to differ between frozen and lyophilized FMT product after 9, 11, or 15 months [27]. An aliquot of the final fecal filtrate will be stored at 
- $80{ }^{\circ} \mathrm{C}$ for analysis in case retroactive testing of the fecal filtrate is required.

\section{FMT delivery and mucosal biopsies}

Prior to colonoscopy, three falcon tubes of frozen fecal filtrate, corresponding to a total of $150 \mathrm{~g}$ original donor feces, will be thawed at room temperature over $2.5 \mathrm{~h}$. Thawed feces will be diluted to a total volume of 300 $\mathrm{mL}$ with $0.9 \mathrm{~N} \mathrm{NaCl}$ and packaged into $6 \times 50 \mathrm{~mL}$ syringes for colonoscopy. The evening prior to colonoscopy, study subjects will take $4 \mathrm{~L}$ of polyethylene glycol laxative solution. Colonoscopy will be performed by an experienced gastroenterologist at the University Health Network. First, six mucosal pinch biopsies ( $3 \mathrm{~mm}$ size) will be taken: two each from the end of the rectum $(20 \mathrm{~cm}$ from the anal verge), the ascending colon, and the terminal ileum. These samples will be used for compositional and metabolomic profiling of the mucosaadherent microbiome. Biopsies will also be sent to pathology if clinically needed. Second, the gastroenterologist will collect $5 \mathrm{~mL}$ of colon secretion into sterile microtubes (secretions will be collected via trap). This will be frozen immediately and stored at $-80{ }^{\circ} \mathrm{C}$ and used to test for pathogens at the time of FMT in case a recipient develops an infectious disease. Finally, participants will be placed in the right lateral recumbency position, and FMT will be performed with fecal filtrate in one syringe deposited in the terminal ileum and five in the cecum.

\section{Data collection, timeline, and outcomes Outcomes}

The study visit schedule is detailed in Table 1. A description of the tools used to measure specific outcomes is provided below. The primary outcome measures for the pilot study are (1) feasibility, (2) acceptability, and (3) adherence with the trial protocol.

Table 1 Study schedule for the participants in the pilot randomized controlled trial of fecal microbiota transplantation (FMT) treatment of bipolar depression

\begin{tabular}{|c|c|c|c|c|c|c|}
\hline \multirow[t]{3}{*}{ Measure } & \multicolumn{5}{|c|}{ Study period } & \multirow[t]{3}{*}{ Purpose } \\
\hline & \multicolumn{2}{|c|}{$\begin{array}{l}\text { Prior to } \\
\text { intervention }\end{array}$} & \multirow{2}{*}{$\begin{array}{l}\text { Intervention } \\
\text { Colonoscopy } \\
\text { visit }\end{array}$} & \multicolumn{2}{|c|}{ Follow-up (12 visits every 2 weeks) } & \\
\hline & Screening & Baseline & & $\begin{array}{l}\text { Every } 2 \\
\text { weeks }\end{array}$ & $\begin{array}{l}\text { Week } 12 \text { and week } 24 \\
\text { visits }\end{array}$ & \\
\hline Eligibility screening & $\mathrm{x}$ & & & & & Feasibility \\
\hline Informed consent & $x$ & & & & & Feasibility \\
\hline Confirmation of eligibility & & $\mathrm{x}$ & & & & Feasibility \\
\hline Randomization & & $x$ & & & & Feasibility \\
\hline Colonoscopy & & & $\mathrm{X}$ & & & Intervention \\
\hline Anthropometric measurements & & $x$ & & $\mathrm{x}$ & & Covariates \\
\hline Food diary & & $\mathrm{x}$ & & & $\mathrm{x}$ & Covariates \\
\hline Mucosa biopsies/colon secretion & & & $\mathrm{x}$ & & & $\begin{array}{l}\text { Microbial } \\
\text { analysis }\end{array}$ \\
\hline Stool sample & & $\mathrm{x}$ & & & $x$ & $\begin{array}{l}\text { Microbial } \\
\text { analysis }\end{array}$ \\
\hline Blood sample & & & $\mathrm{x}$ & & & Safety \\
\hline Concomitant medications & $x$ & $\mathrm{x}$ & $\mathrm{x}$ & $\mathrm{x}$ & & Safety \\
\hline Symptoms diary & & & & $\mathrm{x}$ & & $\begin{array}{l}\text { Safety/ } \\
\text { tolerance }\end{array}$ \\
\hline Toronto Side Effect Scale (TSES) & & $\mathrm{x}$ & & $\mathrm{x}$ & & Safety/efficacy \\
\hline $\begin{array}{l}\text { Mini-International Neuropsychiatric Interview } \\
\text { (MINI) }\end{array}$ & $\mathrm{x}$ & & & & & Feasibility \\
\hline Young Mania Rating Scale (YMRS) & $x$ & $\mathrm{x}$ & & $\mathrm{x}$ & & Efficacy \\
\hline $\begin{array}{l}\text { Montgomery-Asberg Depression Rating Scale } \\
\text { (MADRS) }\end{array}$ & $\mathrm{x}$ & $\mathrm{X}$ & & $\mathrm{x}$ & & Efficacy \\
\hline State and Trait Anxiety Inventory (STAI) & & $\mathrm{x}$ & & $\mathrm{x}$ & & Efficacy \\
\hline Sheehan Disability Scale (SDS) & & $\mathrm{x}$ & & $\mathrm{x}$ & & Efficacy \\
\hline $\begin{array}{l}\text { World Health Organization Quality of Life } \\
\text { (WHOQOL-BREF) }\end{array}$ & & $\mathrm{x}$ & & $\mathrm{x}$ & & Efficacy \\
\hline
\end{tabular}


Feasibility, clinical, tolerability, and safety outcomes We will record feasibility data related to (i) eligibility (for example, proportion of mental health clinic patients eligible), (ii) recruitment (for example, number, nonparticipation reasons, especially the decision related to the colonoscopy aspect of the study), and (iii) timing (for example, time before participant begins treatment).

Safety of FMT will be assessed from unsolicited and solicited adverse events. Clinical and tolerability scores at baseline (Visit 2) will be compared with those at study completion (Visit 15). In addition, the Toronto Side Effects Scale (TSES) [28], a 32-item instrument designed to establish incidence, frequency, and severity of CNS, gastrointestinal, and sexual side effects will be used.

Assessment tools The following outcome measures will be used to assess symptoms: the MADRS [19], a 10-item questionnaire which psychiatrists use to measure the severity of depressive episodes in patients with mood disorders; the YMRS [20], which is 11 items and is a clinician-administered interview scale used to assess manic symptoms; the State Trait Anxiety Index (STAI) [29], a commonly used measure of anxiety that can be used to diagnose anxiety and to distinguish it from depressive syndromes; the Clinical Global Impressions (CGI) rating scales [30], commonly used to measure symptom severity and treatment response in studies of patients with mental disorders; and the Sheehan Disability Scale (SDS) [31], a brief self-report tool developed to assess functional impairment in work/school, social life, and home life or family responsibilities domains. The World Health Organization Brief Quality of Life Scale (WHOQOL-BREF) [32], a quality of life assessment designed to be applicable cross-culturally, will also be administered.

Microbiome composition Fecal microbiome profiling will be performed using Illumina sequencing. DNA extraction will be performed using a previously described protocol that enhances DNA recovery from microbial communities [33, 34], with modifications [35]. Total eubacterial load will be measured via Quantitative polymerase chain reaction (qPCR) with universal primers 8fM and Bact515R [36]. Thermal cycle reactions will run on the Bio-Rad CFX96 Real-Time System. Samples will be prepared in triplicates and quantified based on a standard curve with an efficiency of $94.9 \%$ generated by 10-fold serial dilutions of a plasmid containing a single copy of the 16S rRNA gene. The negative control includes a no template reaction to control for reagent contamination.

Bacterial community profiling of the 16S rRNA gene will be performed using paired-end reads of the V3 region using bar-coded Illumina sequencing as described previously [37] with bar codes included in the forward primer. Alternatively, amplification of the $\mathrm{V} 3-\mathrm{V} 4$ region will be performed using 806R primer [38]. Amplified product will be purified by separation via agarose gel electrophoresis and gel extraction. Pair-end sequencing will be performed on a MiSeq Illumina sequencer as per manufacturer's instructions, as described previously [39].

Microbiome metabolomics Fecal samples will be collected and stored at $-80^{\circ} \mathrm{C}$, and fecal supernatants will be analyzed via NMR spectrometry [40].

Mucosal biopsy analysis Of the two biopsies from each of the three locations, one will be stored in $10 \%$ formalin for pathological assessment and the other stored at -80 ${ }^{\circ} \mathrm{C}$ for microbiome analysis. DNA will be extracted from $3 \mathrm{mg}$ of tissue and purified as for the stool. Sequencing and analysis will be performed as for stool samples.

A baseline visit with the research coordinator (blind to group allocation) occurs in person, prior to the start of the active study phase, to collect baseline demographic and health service use information as well as baseline measures on mental health symptom scales. During all subsequent visits (visit 1 to visit 15), concomitant medications will be recorded. Anthropometric measurements (waist-to-hip ratio, and body mass index (BMI)) will be taken at every visit, except during the first visit for screening (visit 2-visit 15). Food intake will be recorded for 3 days ( 2 weekdays and 1 weekend day), and the completed forms will be collected during visit 2 (baseline), 9 (12 weeks), and 15 (24 weeks). To minimize confounding effects, participants will be asked to maintain their usual diet and exercise level. Participants will also itemize their food intake using a validated form developed by the Fred Hutchinson Cancer Research Center [41]. Food records will be readministered at 3- and 6month time points. Dietary data will be analyzed using NUTRITIONIST PRO ${ }^{\text {tw }}$ DIET ANALYSIS SOFTWARE (Axxya Systems) to assess intake of macro- and micronutrients.

Pre-intervention assessments At Visit 1, informed written consent will be obtained, and demographic characteristics, current medical conditions and concurrent medications will be recorded. The following questionnaires will also be administered: MINI [17] for diagnosis of BD, YMRS [20] for mania, and MADRS for depression [19]. Recipients will receive instructions and two kits for collecting two stool samples at home, and a standard food record to measure dietary intake for 3 days. As diet and bowel preparation alter the microbiome, stool samples will be collected at Visit 2, 3 to 4 days prior to colonoscopy. A fraction of these fecal samples will be stored at $-80{ }^{\circ} \mathrm{C}$ for microbiome analysis. 
After randomization, $150 \mathrm{~g}$ of the remaining, refrigerated feces will be manufactured into FMT product. A portion of FMT fecal filtrate will be frozen at $-80{ }^{\circ} \mathrm{C}$ for future analysis, and $20 \mathrm{~mL}$ of blood will also be collected prior to randomization for analysis if a recipient is later found to be infected with a transmissible disease. For baseline clinical scores, the following questionnaires will be given: MADRS [19], YMRS [20], CGI [30], STAI [42], SDS [31], WHOQOL-BREF [32], and the TSES [28].

Intervention visit At Visit 3, FMT will be performed. To document side effects, patients will be provided with a symptom diary after FMT, which they will submit at every subsequent study visit.

Post-intervention visits To evaluate whether FMT effects longer-term changes in depression severity and microbiome profile, patients will be followed for 6 months (24 weeks) post-FMT, during Visits 3-15. Clinical (MADRS, YMRS, CGI, STAI, SDS, WHOQOLBREF) and tolerability (TSES) outcomes will be assessed every 2 weeks after FMT for 6 months. Adverse events will be verbally screened for biweekly. Stool samples will be collected at 3 and 6 months to assess fecal microbiome composition post-FMT.

\section{Statistical methods}

We will use descriptive methods to estimate feasibility and compliance with the FMT intervention, the number of FMT and placebo treatments, the number of fecal samples provided, the duration of follow-up, recruitment rate, rates of nonparticipation, and so on. We will calculate acceptability using reported side effects and patient outcomes.

Variable distribution will be assessed using graphical methods and descriptive statistics, including means, standard deviations, medians and 1st and 3rd quartiles, frequencies and proportions, will be calculated where appropriate. Randomized groups will be compared to assess whether imbalance in covariates such as age and gender are present. For continuous measures (e.g., MADRS scores), change scores will be calculated as the difference between baseline and 6-month visit assessments. Groups will be compared using independent samples $t$ test or Wilcoxon signed rank test as appropriate. For binary measures (e.g., proportion of side effects), the difference between groups will be tested using Chisquare or Fisher exact test as appropriate. Bivariate relationships between outcome measures and changes in microbiome composition (e.g., relative abundances of taxa of interest) will be explored using Spearman and Pearson correlation coefficients. For microbiome and metabolome data, multivariate dimensionality reduction approaches, such as Principal Components analysis and cluster analysis, will be employed.

Trajectory plots will be created to examine changes in outcome measures over time. Generalized linear regression models will then be used to assess differences [43] between groups at different time points, as was well as to explore the relationship between these changes and microbiome characteristics. In order to account for correlation between time points, random effects models and generalized estimating equations approach will be used. All models will include group, time, and group by time interaction terms; additionally, factors with known influence on outcome measures will be considered for inclusion as covariates, particularly if found imbalanced between the randomized groups. For patient dropout, the missingness patterns will be assessed, and appropriate methods of handling missing data will be used [44]. Goodness-of-fit will be applied for model diagnostics.

Statistical analyses will be performed using SAS 9.4, R 3.1, as well as analytic tools available in QIIME package. All tests will be two-sided with a significance level of $5 \%$. Where necessary, $p$ value adjustments for multiple testing (e.g., Bonferroni, Holm's Bonferroni Step-down, Benjamini and Hochberg False Discovery Rate) will be employed.

\section{Sample size}

This is a pilot feasibility trial designed to show if a larger RCT trial would be worthwhile. Therefore, no formal sample size calculation has been carried out [45]. A review by Hertzog suggests a range of 20 to 40 participants to allow for sufficient variability in acceptability assessment of an intervention [46]. Based on work from a pharmaceutical study in a similar patient population [47], the expected standard deviation of change expected in the MADRS score is approximately 12 . Therefore, with 25 patients per group, we will be able to quantify the difference in change in MADRS score between groups to within 6.5 points with $95 \%$ confidence. This will allow data that can be used in the generation of a sample size for a larger trial. To account for a 15\% dropout rate, we plan to recruit 60 patients; 30 will receive the active treatment (allogenic FMT), and 30 will receive the control treatment (autologous FMT).

\section{Data management and monitoring Data monitoring and auditing}

A Data Monitoring Committee will identify mechanisms to ensure the safety and efficacy of all aspects of the study. This committee will review the research protocol, informed consent documents, plans for data and safety monitoring, and steps taken to maintain patient data confidentiality. Furthermore, the committee will monitor all stages of the study, including factors that may 
influence study quality (data quality and timeliness, participant recruitment, accrual and retention, participant risk versus benefit); consider new scientific or therapeutic evidence with implications for the safety and ethicality of the study; and review serious adverse event documentation and safety reports. Adverse events will be assessed verbally. Additionally, patients will be provided with a symptom diary covering symptoms that may indicate an adverse event related to FMT in the 7 days following the intervention. A physician will be responsible for all trial-related medical decisions and review of adverse events and severe adverse events throughout the study. All unanticipated problems will be reported to the local and main REBs. Adverse drug reactions (adverse events related or potentially related to the study project that are both serious and unexpected) will be subject to expedited reporting to Health Canada.

\section{Dissemination}

Results will be published in scientific journals and presented at research conferences. We will also share our results via the "Gut Microbiota for Health Experts exchange," an online community where experts in microbiome and health-related fields share news, innovation, and information on topics pertaining to the gut microbiota. The results will also be made available to individuals suffering from BD, who are public stakeholders in the study, via advocacy groups.

\section{Discussion}

Bipolar disorder is a common and disabling mental illness with significant morbidity and mortality. People living with $\mathrm{BD}$ experience substantial impairment, being symptomatic with syndromal or subsyndromal symptoms, particularly those of depression, for approximately half of their lives [48]. It is chronic and highly recurrent, with close to $60 \%$ of the patients experiencing a recurrence of $\mathrm{BD}$ in the first 2 years, and about $75 \%$ experiencing a recurrence in over 5 years following the initial diagnosis [49]. Reoccurrence often occurs in the face of treatment noncompliance, which is due in part to the significant side effects associated with $\mathrm{BD}$ treatments [50], but it can also occur when there is adherence to a treatment plan [51]. There is therefore a need to locate treatments with better tolerability and efficacy. Compelling evidence supports an MGB-axis link and as a recent review concluded, there is growing support for the treatment and transmission of psychiatric illnesses through FMT. Further research with stronger scientific design is warranted in order to fully determine the efficacy and safety of this potential treatment [15].

The strengths of our protocol include (1) the use of a novel treatment option for BD depression that has not been previously described in this population; (2) a strong interdisciplinary team of coinvestigators to ensure participant safety via the use of well screened and processed FMT; (3) extensive follow-up, to monitor depressive symptoms 6 months after the intervention; (4) study oversight by a strong team with a diverse range of expertise and training in the fields of neuroscience, gastroenterology, infectious disease, and microbiology; and (5) a comprehensive dissemination plan to ensure adequate uptake of the knowledge that this study will generate. There are some limitations to the pilot study methodology. First, we restricted our sample recruitment to those who failed previous treatments. Thus, the effect of FMT within our treatment-resistant population may not represent its effect in a nonresistant population. Second, we excluded those who had gastrointestinal issues such as IBD, conditions which could alter outcomes of FMT. The nature of the colonoscopy intervention may also result in particular patient selection, excluding those medically unable to tolerate this procedure. Finally, because this is a pilot study, our results will not support definitive conclusions on efficacy or safety of FMT in BD depression but may help guide the development of a larger study to focus on these outcomes.

In summary, this protocol was developed through strong collaborations between psychiatry, gastroenterology, and infectious disease delivery providers and research practices and represents a multisite hospital collaboration. This pilot RCT will allow us to assess the feasibility of novel treatments for BD depression and to understand potential mechanisms of how microbial manipulation can impact disease outcomes. This will help us guide the development of larger trials in this area.

\section{Study status}

Participant enrollment began February 1st, 2017. We have currently enrolled 25 participants.

\section{Abbreviations}

BD: Bipolar disorder; FMT: Fecal microbiota transplantation; MGB: Microbiota - gut-brain; CNS: Central nervous system; RCT: Randomized controlled trial; MINI: Mini International Neuropsychiatric Interview; MADRS: MontgomeryAsberg Depression Rating Scale; YMRS: Young Mania Rating Scale; MTOP: Microbial Therapeutics Outcome Program; STAI: The State Trait Anxiety Index; CGI: Clinical Global Impressions; SDS: Sheehan Disability Scale; WHOQOL-BREF: World Health Organization Brief Quality of Life Scale; qPCR: Quantitative polymerase chain reaction; NMR: Nuclear magnetic resonance; BMI: Body mass index

\section{Authors' contributions}

$\mathrm{VT}$ is the primary investigator of the study and developed the study design and trial schema. She drafted the manuscript, edited it, and approved the final version for publication. $\mathrm{AB}, \mathrm{JA}, \mathrm{SH}$, and $\mathrm{SP}$ contributed to the development of the study design and trial schema, edited the manuscript, and approved the final version for publication. NCAC helped draft and edit the manuscript. The authors read and approved the final manuscript

\section{Funding}

This clinical trial was supported by a The Stanley Medical Research Institute (SMRI) grant (15T-009). The funder played no role in the design of the study 
and collection, analysis, and interpretation of data and in writing the manuscript.

\section{Availability of data and materials}

The datasets used and/or analyzed during the current study are available from the corresponding author on reasonable request.

\section{Declarations}

\section{Ethics approval and consent to participate}

This study was conducted in accordance with the Declaration of Helsinki and was approved by Health Canada on January 13, 2017. The study was reviewed and approved by the Women's College Hospital Ethics board (2017-0099-B), The Mount Sinai Hospital Research Ethics Board (17-0289-E), The University Health Network Research Ethics Board (17-5147), and the Canadian SHIELD Ethics Review Board (2020-09-001). Written informed consent to participate was obtained from each patient before enrollment in the study. This study has been registered in the Clinicaltrial.gov (https:// www.clinicaltrials.gov/ct2/show/NCT03279224) on 12 September 2017.

\section{Consent for publication}

Not applicable

\section{Competing interests}

The principal investigator and site investigators have no competing interests to declare. The funding agencies had no role in the conduct of the study.

\section{Author details}

${ }^{1}$ Snyder Institute for Chronic Diseases, Cumming School of Medicine, University of Calgary, Calgary, Alberta, Canada. ${ }^{2}$ Department of Psychiatry, Cumming School of Medicine, University of Calgary, Calgary, Alberta, Canada. ${ }^{3}$ Department of Medicine, Toronto General Hospital, University of Toronto, Toronto, Ontario, Canada. ${ }^{4}$ Infection Prevention and Control Department, Department of Medicine, University of Toronto, Toronto, Ontario, Canada. ${ }^{5}$ Departments of Microbiology and Medicine, University Health Network and Sinai Health, University of Toronto, Toronto, Canada. ${ }^{6}$ Hotchkiss Brain Institute, Cumming School of Medicine, University of Calgary, Calgary, Alberta, Canada.

\section{Received: 20 March 2021 Accepted: 7 July 2021}

Published online: 14 July 2021

\section{References}

1. Merikangas KR, Akiskal HS, Angst J, Greenberg PE, Hirschfeld RM, Petukhova $M$, et al. Lifetime and 12-month prevalence of bipolar spectrum disorder in the National Comorbidity Survey replication. Arch Gen Psychiatry. 2007; 64(5):543-52. https://doi.org/10.1001/archpsyc.64.5.543.

2. Forte A, Baldessarini RJ, Tondo L, Vazquez GH, Pompili M, Girardi P. Long-term morbidity in bipolar-I, bipolar-II, and unipolar major depressive disorders. J Affect Disord. 2015;178:71-8. https://doi.org/10.1016/j.jad.2015.02.011.

3. Tondo L, Vazquez GH, Baldessarini RJ. Options for pharmacological treatment of refractory bipolar depression. Current psychiatry reports. 2014; 16(2):431. https://doi.org/10.1007/s11920-013-0431-y.

4. Butler M, Urosevic S, Desai P, Sponheim SR, Popp J, Nelson VA, et al. AHRQ comparative effectiveness reviews. In: Treatment for Bipolar Disorder in Adults: A Systematic Review. Rockville (MD): Agency for Healthcare Research and Quality (US; 2018.

5. Forsythe P, Sudo N, Dinan T, Taylor VH, Bienenstock J. Mood and gut feelings. Brain Behav Immun. 2010;24(1):9-16. https://doi.org/10.1016/j.bbi.2009.05.058.

6. Evrensel A, Ceylan ME. The gut-brain axis: the missing link in depression. Clin Psychopharmacol Neurosci. 2015;13(3):239-44. https://doi.org/10.9758/ cpn.2015.13.3.239.

7. Hu S, Li A, Huang T, Lai J, Li J, Sublette ME, et al. Gut microbiota changes in patients with bipolar depression. Adv Sci (Weinh). 2019;6(14):1900752 https://doi.org/10.1002/advs.201900752

8. Coello K, Hansen TH, Sorensen N, Munkholm K, Kessing LV, Pedersen O, et al. Gut microbiota composition in patients with newly diagnosed bipolar disorder and their unaffected first-degree relatives. Brain Behav Immun. 2019;75:112-8. https://doi.org/10.1016/j.bbi.2018.09.026.

9. Painold A, Morkl S, Kashofer K, Halwachs B, Dalkner N, Bengesser S, et al. A step ahead: exploring the gut microbiota in inpatients with bipolar disorder during a depressive episode. Bipolar Disord. 2019;21(1):40-9. https://doi. org/10.1111/bdi.12682

10. Gondalia S, Parkinson L, Stough C, Scholey A. Gut microbiota and bipolar disorder: a review of mechanisms and potential targets for adjunctive therapy. Psychopharmacology. 2019;236(5):1433-43. https://doi.org/10.1007/ s00213-019-05248-6.

11. Evans SJ, Bassis CM, Hein R, Assari S, Flowers SA, Kelly MB, et al. The gut microbiome composition associates with bipolar disorder and illness severity. J Psychiatr Res. 2017;87:23-9. https://doi.org/10.1016/j.jpsychires.2016.12.007.

12. Wallace CJK, Milev R. The effects of probiotics on depressive symptoms in humans: a systematic review. Ann General Psychiatry. 2017;16(1):14. https:// doi.org/10.1186/s12991-017-0138-2.

13. Yulug B, Schabitz WR. Towards a multimodal treatment of depression: a minireview on the potential role of antibiotics. Mini-Rev Med Chem. 2011 11(11):984-7. https://doi.org/10.2174/138955711797068418.

14. Weingarden AR, Vaughn BP. Intestinal microbiota, fecal microbiota transplantation, and inflammatory bowel disease. Gut Microbes. 2017:8(3): 238-52. https://doi.org/10.1080/19490976.2017.1290757.

15. Chinna Meyyappan A, Forth E, Wallace CJK, Milev R. Effect of fecal microbiota transplant on symptoms of psychiatric disorders: a systematic review. BMC Psychiatry. 2020;20(1):299. https://doi.org/10.1186/s12888-02002654-5.

16. Chan AW, Tetzlaff JM, Altman DG, Laupacis A, Gotzsche PC, Krleza-Jeric K, et al. SPIRIT 2013 statement: defining standard protocol items for clinical trials. Ann Intern Med. 2013;158(3):200-7. https://doi.org/10.7326/0003-481 9-158-3-201302050-00583.

17. Sheehan DV, Lecrubier $Y$, Sheehan $\mathrm{KH}$, Amorim $\mathrm{P}$, Janavs J, Weiller $\mathrm{E}$, et al. The Mini-International Neuropsychiatric Interview (M.I.N.I.): the development and validation of a structured diagnostic psychiatric interview for DSM-IV and ICD-10. The Journal of clinical psychiatry. 1998:59(Suppl 20):22-33 quiz 34-57.

18. Yatham LN, Kennedy SH, Parikh SV, Schaffer A, Bond DJ, Frey BN, et al. Canadian Network for Mood and Anxiety Treatments (CANMAT) and International Society for Bipolar Disorders (ISBD) 2018 guidelines for the management of patients with bipolar disorder. Bipolar Disord. 2018;20(2): 97-170. https://doi.org/10.1111/bdi.12609.

19. Montgomery SA, Asberg M. A new depression scale designed to be sensitive to change. Br J Psychiatry. 1979:134(4):382-9. https://doi.org/10.11 92/bjp.134.4.382

20. Young RC, Biggs JT, Ziegler VE, Meyer DA. A rating scale for mania: reliability, validity and sensitivity. Br J Psychiatry. 1978;133(11):429-35. https://doi.org/10.1192/bjp.133.5.429.

21. Bell CC. DSM-IV: Diagnostic and Statistical Manual of Mental Disorders. JAMA. 1994:272(10):828-9. https://doi.org/10.1001/jama.1994.03520100096046.

22. Drago L, Toscano M, De Grandi R, Casini V, Pace F. Persisting changes of intestinal microbiota after bowel lavage and colonoscopy. Eur J Gastroenterol Hepatol. 2016:28(5):532-7. https://doi.org/10.1097/MEG. 0000000000000581

23. Jalanka J, Salonen A, Salojarvi J, Ritari J, Immonen O, Marciani L, et al. Effects of bowel cleansing on the intestinal microbiota. Gut. 2015;64(10):1562-8. https://doi.org/10.1136/gutjnl-2014-307240.

24. Hamilton MJ, Weingarden AR, Sadowsky MJ, Khoruts A. Standardized frozen preparation for transplantation of fecal microbiota for recurrent Clostridium difficile infection. Am J Gastroenterol. 2012;107(5):761-7. https://doi.org/10.1 038/ajg.2011.482.

25. Hamilton MJ, Weingarden AR, Unno T, Khoruts A, Sadowsky MJ. Highthroughput DNA sequence analysis reveals stable engraftment of gut microbiota following transplantation of previously frozen fecal bacteria. Gut Microbes. 2013;4(2):125-35. https://doi.org/10.4161/gmic.23571.

26. Costello SP, Conlon MA, Vuaran MS, Roberts-Thomson IC, Andrews JM. Faecal microbiota transplant for recurrent Clostridium difficile infection using longterm frozen stool is effective: clinical efficacy and bacterial viability data. Aliment Pharmacol Ther. 2015;42(8):1011-8. https://doi.org/10.1111/apt.13366.

27. Jiang ZD, Alexander A, Ke S, Valilis EM, Hu S, Li B, et al. Stability and efficacy of frozen and lyophilized fecal microbiota transplant (FMT) product in a mouse model of Clostridium difficile infection (CDI). Anaerobe. 2017:48:1104. https://doi.org/10.1016/j.anaerobe.2017.08.003

28. Vanderkooy JD, Kennedy SH, Bagby RM. Antidepressant side effects in depression patients treated in a naturalistic setting: a study of bupropion, moclobemide, paroxetine, sertraline, and venlafaxine. Can J Psychiatr. 2002: 47(2):174-80. https://doi.org/10.1177/070674370204700208. 
29. Spielberger CD. State-trait anxiety inventory: bibliography, 2nd Edition edn. Palo Alto, CA: Consulting Psychologists Press; 1989.

30. Busner J, Targum SD. The clinical global impressions scale: applying a research tool in clinical practice. Psychiatry (Edgmont). 2007;4(7):28-37.

31. Sheehan DV, Harnett-Sheehan K, Raj BA. The measurement of disability. Int Clin Psychopharmacol. 1996;11(Suppl 3):89-95. https://doi.org/10.1097/ 00004850-199606003-00015.

32. Development of the World Health Organization WHOQOL-BREF quality of life assessment. The WHOQOL Group. Psychol Med. 1998;28(3):551-8.

33. Sibley CD, Parkins MD, Rabin HR, Duan K, Norgaard JC, Surette MG. A polymicrobial perspective of pulmonary infections exposes an enigmatic pathogen in cystic fibrosis patients. Proc Natl Acad Sci U S A. 2008;105(39): 15070-5. https://doi.org/10.1073/pnas.0804326105.

34. Sibley CD, Grinwis ME, Field TR, Eshaghurshan CS, Faria MM, Dowd SE, et al. Culture enriched molecular profiling of the cystic fibrosis airway microbiome. PLoS One. 2011;6(7):e22702. https://doi.org/10.1371/journal. pone.0022702.

35. Whelan FJ, Verschoor CP, Stearns JC, Rossi L, Luinstra K, Loeb M, et al. The loss of topography in the microbial communities of the upper respiratory tract in the elderly. Ann Am Thorac Soc. 2014;11(4):513-21. https://doi.org/1 0.1513/AnnalsATS.201310-3510C.

36. Nadkarni MA, Martin FE, Jacques NA, Hunter N. Determination of bacterial load by real-time PCR using a broad-range (universal) probe and primers set. Microbiology (Reading). 2002;148(Pt 1):257-66. https://doi.org/10.1099/ 00221287-148-1-257.

37. Bartram AK, Lynch MD, Stearns JC, Moreno-Hagelsieb G, Neufeld JD. Generation of multimillion-sequence 16S rRNA gene libraries from complex microbial communities by assembling paired-end illumina reads. Appl Environ Microbiol. 2011;77(11):3846-52. https://doi.org/1 0.1128/AEM.02772-10.

38. Relman DA, Loutit JS, Schmidt TM, Falkow S, Tompkins LS. The agent of bacillary angiomatosis. An approach to the identification of uncultured pathogens. N Engl J Med. 1990;323(23):1573-80. https://doi.org/10.1056/ NEJM199012063232301.

39. Stearns JC, Lynch MD, Senadheera DB, Tenenbaum HC, Goldberg MB, Cvitkovitch DG, et al. Bacterial biogeography of the human digestive tract. Sci Rep. 2011;1(1):170. https://doi.org/10.1038/srep00170.

40. Psychogios N, Hau DD, Peng J, Guo AC, Mandal R, Bouatra S, et al. The human serum metabolome. PLoS One. 2011;6(2):e16957. https://doi.org/1 0.1371/journal.pone.0016957.

41. Kwan ML, Kushi LH, Song J, Timperi AW, Boynton AM, Johnson KM, et al. A practical method for collecting food record data in a prospective cohort study of breast cancer survivors. Am J Epidemiol. 2010;172(11):1315-23. https://doi.org/10.1093/aje/kwq284.

42. Spielberger CD. State-trait anxiety inventory: bibliography. 2nd ed. Palo Alto, CA: Consulting Psychologists Press; 1998.

43. Hedeker DR, Gibbons RD. Longitudinal data analysis. Hoboken, N.J: WileyInterscience; 2006

44. Diggle P, Zeger SL, Liang KY, Heagerty P. Analysis of longitudinal data. Oxford, England: Oxford University Press; 2002.

45. Thabane L, Ma J, Chu R, Cheng J, Ismaila A, Rios LP, et al. A tutorial on pilot studies: the what, why and how. BMC Med Res Methodol. 2010;10(1):1. https://doi.org/10.1186/1471-2288-10-1.

46. Hertzog MA. Considerations in determining sample size for pilot studies. Res Nurs Health. 2008;31(2):180-91. https://doi.org/10.1002/nur.20247.

47. Suppes $T$, Dennehy EB, Hirschfeld RM, Altshuler LL, Bowden CL, Calabrese JR, et al. The Texas implementation of medication algorithms: update to the algorithms for treatment of bipolar I disorder. The Journal of clinical psychiatry. 2005;66(7):870-86. https://doi.org/10.4088/ JCP.v66n0710.

48. Judd LL, Akiskal HS, Schettler PJ, Endicott J, Maser J, Solomon DA, et al. The long-term natural history of the weekly symptomatic status of bipolar I disorder. Arch Gen Psychiatry. 2002;59(6):530-7. https://doi.org/10.1001/a rchpsyc.59.6.530.

49. Gitlin MJ, Swendsen J, Heller TL, Hammen C. Relapse and impairment in bipolar disorder. Am J Psychiatry. 1995;152(11):1635-40. https://doi.org/10.11 76/ajp.152.11.1635

50. Gaudiano BA, Weinstock LM, Miller IW. Improving treatment adherence in bipolar disorder: a review of current psychosocial treatment efficacy and recommendations for future treatment development. Behav Modif. 2008;32(3):267-301. https://doi.org/10.11 77/0145445507309023.

51. Albuquerque J, Deshauer D, Fergusson D, Doucette S, MacWilliam C, Kaufmann IM. Recurrence rates in Ontario physicians monitored for major depression and bipolar disorder. Can J Psychiatr. 2009;54(11):777-82. https:// doi.org/10.1177/070674370905401108.

\section{Publisher's Note}

Springer Nature remains neutral with regard to jurisdictional claims in published maps and institutional affiliations.

\section{Ready to submit your research? Choose BMC and benefit from:}

- fast, convenient online submission

- thorough peer review by experienced researchers in your field

- rapid publication on acceptance

- support for research data, including large and complex data types

- gold Open Access which fosters wider collaboration and increased citations

- maximum visibility for your research: over $100 \mathrm{M}$ website views per year

At BMC, research is always in progress.

Learn more biomedcentral.com/submissions 